\title{
ON THE FAILURE RATE ESTIMATION OF THE INVERSE GAUSSIAN DISTRIBUTION
}

\author{
ZHENLIN YANG and RONNIE T. C. LEE \\ Department of Statistics and Applied Probability, National University of Singapore, \\ 3 Science Drive 2, Singapore 117543.
}

New estimators of the inverse Gaussian failure rate are proposed based on the maximum likelihood predictive densities derived by Yang (1999). These estimators are compared, via Monte Carlo simulation, with the usual maximum likelihood estimators of the failure rate and found to be superior in terms of bias and mean squared error. Sensitivity of the estimators against the departure from the inverse Gaussian distribution is studied.

Keywords: Bias; failure rate; inverse Gaussian distribution; maximum likelihood predictive density; relative efficiency; sensitivity.

\section{INTRODUCTION}

The inverse Gaussian (IG) distribution arises as the first passage time distribution of a Brownian motion, hence it is suitable for describing physical phenomena such as the time to fatigue of a metal specimen and the time to failure of a component, etc.. Its probability density function (pdf), denoted by $I G(\mu, \lambda)$, usually takes the following form:

$$
f(y ; \mu, \lambda)=\left(\lambda / 2 \pi y^{3}\right)^{1 / 2} \exp \left[-\lambda(y-\mu)^{2} / 2 \mu^{2} y\right], y>0 ; \mu>0, \lambda>0,
$$

with the mean, variance, skewness and kurtosis of the distribution given by, respectively, $\mu, \mu^{3} / \lambda, 3 \sqrt{\mu / \lambda}$ and $15 \mu / \lambda$. From this we see that the parameter $\phi=\lambda / \mu$ 
determines the shape of the IG distribution and the density is highly skewed for small to moderate values of $\phi$. As $\phi$ increases, the IG tends to normal. The reliability and failure rate functions for (1) have the forms:

$$
\begin{aligned}
R(t ; \mu, \lambda) & =\Phi\left[(\lambda / t)^{1 / 2}(1-t / \mu)\right]-e^{2 \lambda / \mu} \Phi\left[-(\lambda / t)^{1 / 2}(1+t / \mu)\right] \\
r(t ; \mu, \lambda) & =\frac{\left(\lambda / 2 \pi t^{3}\right)^{1 / 2} \exp \left[-\lambda(t-\mu)^{2} / 2 \mu^{2} t\right]}{\Phi\left[(\lambda / t)^{1 / 2}(1-t / \mu)\right]-e^{2 \lambda / \mu} \Phi\left[-(\lambda / t)^{1 / 2}(1+t / \mu)\right]},
\end{aligned}
$$

where $\Phi$ denotes the cumulative density function $(\mathrm{CDF})$ of the standard normal.

The usual estimator of $r(t ; \mu, \lambda)$ is the maximum likelihood estimator (MLE) where the unknown parameter(s) in the function are replaced by their MLEs. Chang (1994) obtained confidence bounds for $r(t ; \mu, \lambda)$ with the 'center curve' (point estimator) being the MLE. The bounds are obtained by converting the confidence intervals for parameters by means of Bonferroni inequality, hence are conservative. The MLE method is known to be simple and easy to implement, but can give rise to estimators with large bias and mean squared error (MSE) when sample size is not large. Yang (1999) derived the maximum likelihood predictive densities (MLPDs) for the inverse Gaussian distribution and found successful applications in reliability and lifetime predictions. In this article, we consider applying these MLPDs to give estimators of $r(t ; \mu, \lambda)$ for the cases of one or both parameters unknown, and using Monte Carlo simulation to evaluate the performance of these estimators. In the case of lack of information on the relevant distribution, a nonparametric method described in Modarres (1993, p82) can be used, but again this method does not usually have a satisfactory performance when sample size is not large. For a complete account of the IG distribution, see the specialized monographs of Chhikara and Folks (1989), and Seshadri (1993, 1998).

Section 2 describes the MLPDs for the inverse Gaussian distribution and discusses briefly their large sample properties. Section 3 proposes the MLPD estimators (MLPDE) of $r(t ; \mu, \lambda)$, evaluates their small sample properties using Monte Carlo simulation, and compares them with the usual MLEs. Section 4 conducts a sensitiv- 
ity analysis of the two estimators. Section 5 presents some numerical examples for illustration and further comparison. The results obtained indicate that the MLPDE generally outperforms the usual MLE in terms of bias and MSE. When data has a certain departure from the IG distribution, it is shown that the MLPDE is not very sensitive to this departure and it still generally performs better than the MLE.

\section{MLPDs FOR THE IG DISTRIBUTION}

Let $\mathbf{Y}=\left(Y_{1}, Y_{2}, \ldots, Y_{n}\right)$ be a sample of past observations from an IG distribution with pdf $f(y ; \mu, \lambda)$ and $Y_{n+1}$ or $T$ be a future observation from the same population. The MLPD for $T=Y_{n+1}$ based on $\mathbf{Y}=\mathbf{y}$ is defined by Lejeune and Faulkenberry (1982) as follows:

$$
\hat{f}(t \mid \mathbf{y}) \propto \max _{\theta} f(t ; \theta) f(\mathbf{y} ; \theta)
$$

where $\theta=\mu$ or $\lambda$ or $\theta=(\mu, \lambda)$, depending on the parameter situation. Let $\bar{Y}$ and $\tilde{Y}$ be, respectively, the arithmetic and harmonic means of the past sample, and $\bar{y}$ and $\tilde{y}$ be their observed values. The MLPDs for the inverse Gaussian distribution (Yang, 1999) have the forms:

$$
\begin{aligned}
\tilde{f}(t \mid \mathbf{y}) & =k(\mathbf{y}) t^{-\frac{3}{2}}\left\{\frac{n}{\tilde{y}}+\frac{1}{t}-\frac{(n+1)^{2}}{t+n \bar{y}}\right\}^{-\frac{n+1}{2}} \\
\tilde{f}(t \mid \mathbf{y} ; \mu) & =k(\mathbf{y}, \mu) t^{-\frac{3}{2}}\left\{1+\frac{(t-\mu)^{2}}{n t(\bar{y}+\tilde{y}-2 \mu)}\right\}^{-\frac{n+1}{2}}, \\
\tilde{f}(t \mid \mathbf{y} ; \lambda) & =k(\mathbf{y}, \lambda) t^{-\frac{3}{2}} \exp \left[-\frac{\lambda}{2}\left\{\frac{1}{t}+\frac{n}{\tilde{y}}-\frac{(n+1)^{2}}{t+n \bar{y}}\right\}\right],
\end{aligned}
$$

respectively, for the cases of both $\mu$ and $\lambda$ unknown, only $\lambda$ unknown, and only $\mu$ unknown. The quantities $k(\mathbf{y}), k(\mathbf{y}, \mu)$ and $k(\mathbf{y}, \lambda)$ are the normalizing constants which depend only on the past sample. In (6), write $q=n(\bar{y}+\tilde{y}-2 \mu)$ and define

$$
w=\frac{(t-\mu)^{2}}{q t+(t-\mu)^{2}} .
$$

As $w$ is monotonically decreasing for $t \in[0, \mu]$ and monotonically increasing for $t \in[\mu, \infty)$, integrating $\tilde{f}(t \mid \mathbf{y} ; \mu)$ over $[0, \mu]$ and $[\mu, \infty)$, respectively, by applying 
the transformation and then combining the two integrals for cancelation, one obtains $k(\mathbf{y}, \mu)=\mu / \sqrt{q} \beta(1 / 2, n / 2)$. The MLPD (6) is seen to be the same as the Bayesian predictive density given in Chhikara and Guttman (1982). There are no closed form expressions for the other two constants and hence they have to be found through numerical integrations.

Lejeune and Faulkenberry presented a general theory regarding the consistency of a MLPD. They indicated that the conditions of their theory are satisfied by distributions in the exponential family. Thus, the MLPDs given above are consistent for estimating the IG pdf as the inverse Gaussian distribution belongs to the exponential family. In fact, it is not difficult to verify directly that the MLPDs (5)-(7) are consistent estimators of $f(y ; \mu, \lambda)$. We omit this verification for brevity.

\section{THE MLPDEs OF FAILURE RATE}

The IG failure rate function given in (3) looks very complicated, hence the related inference methods should be also complicated. However, when only point estimation is of concern, one is still able to find simple ways to do so, which may be sufficient for most of the practical purposes if the estimator is 'accurate' enough. The simplest method of estimating $r(t ; \mu, \lambda)$ may be the maximum likelihood method where the unknown parameter(s) in $r(t ; \mu, \lambda)$ are simply replaced by their MLEs. Thus, the resulted MLEs of $r(t ; \mu, \lambda)$ become $\hat{r}(t ; \mu)=r(t ; \mu, \hat{\lambda}(\mu))$ when $\lambda$ is unknown, $\hat{r}(t ; \lambda)=r(t ; \hat{\mu}(\lambda), \lambda)$ when $\mu$ is unknown, and $\hat{r}(t)=r(t ; \hat{\mu}, \hat{\lambda})$ when both $\mu$ and $\lambda$ are unknown, where $\hat{\mu}=\bar{Y}$ and $\hat{\lambda}=1 /\left(\tilde{Y}^{-1}-\bar{Y}^{-1}\right)$ are the MLEs and $\hat{\mu}(\lambda)=\bar{Y}$ and $\hat{\lambda}(\mu)=\mu^{2} /(\bar{Y}+\tilde{Y}-2 \mu)$ are the restricted MLEs, given the other parameter.

As mentioned in the introduction, the MLE method is simple, but can give rise to estimators with large bias and MSE. Thus, a better method is desirable. We propose the so-called MLPD estimator (MLPDE) of $r(t ; \mu, \lambda)$ by considering the MLPD as an estimator of the unknown pdf. Thus, the MLPDEs of the IG failure rate are defined 
as

$$
\tilde{r}(t ; \theta)=\frac{\tilde{f}(t \mid \mathbf{y} ; \theta)}{1-\int_{0}^{t} \tilde{f}(x \mid \mathbf{y} ; \theta) d x},
$$

where the components of $\theta=(\mu, \lambda)$ may not be known all at once. Unlike the MLE, the MLPDE incorporates both the past and future observations in the maximization process, hence should be (at least intuitively) less variable than MLE because of the involvement of more information. It may be of interest to see the behavior of the MLPDE of the IG failure rate relative to the existing estimators. As the MLPDE has close analogy to the MLE, we restrict our comparison between these two estimators.

First, it is obvious that the MLEs of $r(t)=r(t ; \mu, \lambda)$ are consistent as the MLEs of $\mu$ and $\lambda$ are consistent. The MLPDEs are also consistent since the MLPDs are consistent estimators of the true density function. Thus, when sample size is large, the two estimators should behave similarly.

Although consistency is an important feature of an estimator, it is much more important for an estimator to possess good small sample properties. The complicated expressions for the MLE and MLPDEs, especially the latter, may prohibit any analytical study regarding the small sample properties of these estimators. We thus turn to Monte Carlo simulation. The case of both parameters unknown is clearly the most practical case. We will concentrate our study on this situation.

We choose four different sample sizes (small to moderate), three combinations of the parameter values that give a population skewness from moderate to large, and six different time points for each combination of sample size and parameter values, which cover more than $80 \%$ of the population values. Each row of the simulation results is based on 10,000 random samples. Simulation results, such as the relative bias and relative efficiency (REF) of MLPDE over MLE, etc., are summarized in Table 1. To give an idea about the standing of each time point in the population, we also calculated the reliabilities at the given time points and listed them in the table under $R(t)$. From the simulation results, we see that the MLPDE can have much smaller 
bias and MSE than the MLE. For example, when $(n, \mu, \lambda, t)=(20,1.0,0.3,1.5)$, we have a $24.2 \%$ relative bias for the MLE compared to a $2.4 \%$ for the MLPDE. The relative efficiency for this case is 2.85, indicating that the MLPDE is much more efficient than the MLE. Increasing the sample size reduces the discrepancy between the two methods. Notice that when $n$ is very small, the MLE can be very unstable. Its MSE can go up to 73.19 (recovered for the values of REF and MSE(MLPDE)), whereas all values for the MSE of the MLPDE are below 1.06. The MLE significantly overestimates the failure rate at the tail part. Both MLE and MLPD agree well with the true value at the early time points. Simulation is also carried out for the case of only one unknown parameter, the results (not reported but available from the authors) show similar features.

Simulation results show that the MLPDE is definitely preferable to the usual MLE. Although the calculation of the MLPDE of the failure rate requires numerical integration, this is not at all a problem as powerful and yet user-friendly statistical softwares, such as Mathematica and Maple, are available. It should be noted that the MLE tends to overestimate the IG failure rate and its relative bias increases with time. It is not clear why this happens. Thus, a general investigation on the analytical behavior of the MLE of failure rate may be of interest.

Although we believe that the behavior of the IG failure rate estimators depends mainly on the shape $(\phi=\lambda / \mu)$ of the distribution, it is important to investigate whether the behavior of the estimators depend on the magnitude of the parameter values. To this end, for $n=30$, we explore the effect of multiplying $(\mu, \lambda)=(.25,1.0)$ by 10 and 100, so that the ratio of $\mu$ and $\lambda$ is kept the same. The simulation results are summarized in Table 2. The results show that the behavior of the estimators does not depend much on the magnitude of the parameters. However, as the parameter values increase, the population becomes more spread out and the values of $r(t)$ thus tend to be smaller. The absolute bias and MSE depend on the magnitude of $r(t)$. 
Table 1: Simulated Biases and MSEs of the Estimators of $r(t), \mu=1.0$.

\begin{tabular}{|c|c|c|c|c|c|c|c|c|c|c|c|}
\hline \multirow[b]{2}{*}{$\lambda$} & \multirow[b]{2}{*}{$t$} & \multirow[b]{2}{*}{$R(t)$} & \multirow[b]{2}{*}{$r(t)$} & \multicolumn{2}{|c|}{ Rel. Bias* } & \multirow[b]{2}{*}{$\mathrm{REF}^{*}$} & \multirow{2}{*}{$\begin{array}{c}\mathrm{MSE} \\
\tilde{r}(t)\end{array}$} & \multicolumn{2}{|c|}{$\overline{\text { Rel. Bias }}$} & \multirow[b]{2}{*}{ REF } & \multirow{2}{*}{$\begin{array}{c}\mathrm{MSE} \\
\tilde{r}(t)\end{array}$} \\
\hline & & & & $\hat{r}(t)$ & $\tilde{r}(t)$ & & & $\hat{r}(t)$ & $\tilde{r}(t)$ & & \\
\hline \multirow{7}{*}{.25} & & & & \multicolumn{4}{|c|}{$n=10$} & \multicolumn{4}{|c|}{$n=20$} \\
\hline & 0.1 & .86 & 2.68 & -4.6 & -7.0 & 1.31 & 1.0551 & -2.7 & -4.4 & 1.17 & 0.5185 \\
\hline & 0.3 & .54 & 1.82 & 18.7 & 7.1 & 1.60 & 0.6158 & 7.5 & 2.1 & 1.24 & 0.1628 \\
\hline & 0.5 & .40 & 1.32 & 30.3 & 11.2 & 2.57 & 0.4430 & 12.7 & 5.1 & 1.41 & 0.1344 \\
\hline & 1.0 & .24 & 0.84 & 48.6 & 8.8 & 11.77 & 0.1687 & 19.7 & 5.3 & 2.04 & 0.0785 \\
\hline & 1.5 & .17 & 0.64 & 63.5 & 0.3 & 47.21 & 0.0676 & 24.2 & 2.4 & 2.85 & 0.0504 \\
\hline & 2.5 & .10 & 0.47 & 101.0 & -15.3 & 2793.4 & 0.0262 & 31.0 & -5.5 & 5.18 & 0.0254 \\
\hline \multirow[t]{6}{*}{1.0} & 0.3 & .83 & 1.29 & -2.7 & -5.5 & 1.26 & 0.2714 & -1.5 & -3.0 & 1.13 & 0.1249 \\
\hline & 0.6 & .55 & 1.36 & 16.4 & 6.4 & 1.47 & 0.3541 & 6.8 & 2.5 & 1.18 & 0.1120 \\
\hline & 0.9 & .38 & 1.24 & 27.3 & 9.4 & 2.23 & 0.3626 & 11.3 & 4.6 & 1.38 & 0.1282 \\
\hline & 1.2 & .26 & 1.14 & 35.2 & 7.1 & 3.77 & 0.2910 & 13.4 & 3.5 & 1.62 & 0.1165 \\
\hline & 1.5 & .19 & 1.06 & 39.3 & 1.9 & 4.77 & 0.2028 & 15.5 & 1.7 & 1.96 & 0.1051 \\
\hline & 2.0 & .11 & 0.96 & 51.6 & -8.1 & 337.4 & 0.1251 & 16.8 & -3.3 & 2.59 & 0.0801 \\
\hline \multirow[t]{6}{*}{4.0} & 0.5 & .89 & 0.93 & -5.8 & -4.4 & 1.26 & 0.1843 & -2.3 & -1.8 & 1.14 & 0.0941 \\
\hline & 0.7 & .69 & 1.52 & 8.0 & 2.2 & 1.33 & 0.4846 & 3.4 & 0.5 & 1.11 & 0.1541 \\
\hline & 1.0 & .41 & 1.97 & 22.5 & 8.8 & 1.79 & 0.8894 & 9.7 & 4.1 & 1.30 & 0.3053 \\
\hline & 1.3 & .23 & 2.15 & 31.0 & 4.1 & 3.45 & 0.8177 & 13.0 & 2.5 & 1.69 & 0.3876 \\
\hline & 1.6 & .11 & 2.23 & 36.4 & -5.1 & 6.34 & 0.6093 & 14.2 & -2.3 & 2.22 & 0.3749 \\
\hline & 2.0 & .05 & 2.27 & 37.3 & -19.0 & 9.30 & 0.5398 & 16.4 & -8.8 & 2.86 & 0.3424 \\
\hline & & & & \multicolumn{4}{|c|}{$\begin{array}{l}n=30 \\
n=3\end{array}$} & \multicolumn{4}{|c|}{$\begin{array}{c}n=50 \\
\end{array}$} \\
\hline \multirow[t]{6}{*}{.25} & 0.1 & .86 & 2.68 & -1.68 & -2.86 & 1.11 & 0.3289 & -1.10 & -1.81 & 1.07 & 0.2045 \\
\hline & 0.3 & .54 & 1.82 & 4.59 & 1.13 & 1.15 & 0.0887 & 2.81 & 0.85 & 1.09 & 0.0436 \\
\hline & 0.5 & .40 & 1.32 & 7.81 & 3.14 & 1.25 & 0.0730 & 4.40 & 1.83 & 1.14 & 0.0347 \\
\hline & 1.0 & .24 & 0.84 & 11.98 & 3.56 & 1.54 & 0.0443 & 6.86 & 2.40 & 1.28 & 0.0241 \\
\hline & 1.5 & .17 & 0.64 & 15.33 & 2.68 & 1.88 & 0.0339 & 8.23 & 1.70 & 1.41 & 0.0179 \\
\hline & 2.5 & .10 & 0.47 & 19.32 & -1.97 & 2.87 & 0.0200 & 10.35 & -0.58 & 1.72 & 0.0121 \\
\hline \multirow[t]{6}{*}{1.0} & 0.3 & .83 & 1.29 & -1.24 & -2.32 & 1.09 & 0.0805 & -0.76 & -1.45 & 1.05 & 0.0451 \\
\hline & 0.6 & .55 & 1.36 & 4.10 & 1.39 & 1.11 & 0.0670 & 2.54 & 0.95 & 1.07 & 0.0369 \\
\hline & 0.9 & .38 & 1.24 & 7.20 & 3.07 & 1.23 & 0.0744 & 4.05 & 1.71 & 1.13 & 0.0415 \\
\hline & 1.2 & .26 & 1.14 & 8.72 & 2.64 & 1.37 & 0.0722 & 5.39 & 1.98 & 1.21 & 0.0408 \\
\hline & 1.5 & .19 & 1.06 & 9.91 & 1.54 & 1.54 & 0.0683 & 5.65 & 1.00 & 1.28 & 0.0401 \\
\hline & 2.0 & .11 & 0.96 & 11.01 & -1.37 & 1.81 & 0.0577 & 6.65 & -0.33 & 1.42 & 0.0360 \\
\hline \multirow[t]{6}{*}{4.0} & 0.5 & .89 & 0.93 & -2.29 & -1.94 & 1.10 & 0.0640 & -1.39 & -1.22 & 1.06 & 0.0377 \\
\hline & 0.7 & .69 & 1.52 & 2.55 & 0.66 & 1.07 & 0.0965 & 1.59 & 0.46 & 1.04 & 0.0541 \\
\hline & 1.0 & .41 & 1.97 & 6.05 & 2.58 & 1.18 & 0.1850 & 3.58 & 1.57 & 1.11 & 0.1012 \\
\hline & 1.3 & .22 & 2.15 & 8.25 & 1.79 & 1.40 & 0.2473 & 4.73 & 1.08 & 1.22 & 0.1418 \\
\hline & 1.6 & .11 & 2.23 & 8.74 & -1.47 & 1.64 & 0.2523 & 5.12 & -0.73 & 1.34 & 0.1580 \\
\hline & 2.0 & .05 & 2.27 & 10.07 & -5.93 & 1.98 & 0.2606 & 5.95 & -3.33 & 1.51 & 0.1699 \\
\hline
\end{tabular}

${ }^{*}$ Rel. Bias $=100(\operatorname{Bias} / r(t)) ;$ REF $=$ MSE(MLE) $/$ MSE(MLPD). 
Thus, as parameter values increase, we see that the absolute bias and MSE decrease, but their relative values are not changed much.

Table 2: Simulation Results for the Cases of Larger Parameter Values

\begin{tabular}{|c|c|c|c|c|c|c|c|c|}
\hline$\mu$ & $\lambda$ & $t$ & $R(t)$ & $r(t)$ & $\begin{array}{l}\text { Rel. } \\
\hat{r}(t)\end{array}$ & $\begin{array}{c}\operatorname{Bias} \\
\tilde{r}(t)\end{array}$ & REF & $\begin{array}{c}\mathrm{MSE} \\
\tilde{r}(t)\end{array}$ \\
\hline \multirow[t]{12}{*}{10} & 2.5 & 0.8 & 0.9017 & 0.2605 & -2.91 & -2.71 & 1.14 & 0.004707 \\
\hline & & 2.0 & 0.6663 & 0.2244 & 2.27 & -0.63 & 1.09 & 0.001161 \\
\hline & & 3.0 & 0.5446 & 0.1817 & 4.74 & 1.26 & 1.15 & 0.000908 \\
\hline & & 6.0 & 0.3532 & 0.1175 & 8.88 & 3.52 & 1.30 & 0.000645 \\
\hline & & 12.0 & 0.2037 & 0.0742 & 13.86 & 3.71 & 1.67 & 0.000423 \\
\hline & & 25.0 & 0.0964 & 0.0468 & 19.15 & -2.08 & 2.72 & 0.000206 \\
\hline & 10.0 & 2.5 & 0.8873 & 0.1168 & -2.39 & -2.34 & 1.11 & 0.000906 \\
\hline & & 6.0 & & 0.13 & 4.47 & 1.74 & 1.12 & 0.000688 \\
\hline & & 9.0 & 0.3750 & 0.1239 & 7.54 & 3.37 & 1.24 & 0.000771 \\
\hline & & 12.0 & 0.2628 & 0.1136 & 8.92 & 2.82 & 1.37 & 0.000745 \\
\hline & & 15.0 & & 0.10 & 10.13 & 1.75 & 1.5 & 0.000689 \\
\hline & & 20.0 & & 0.09 & 11.83 & -0.75 & 1.86 & 0.000597 \\
\hline \multirow[t]{12}{*}{100} & 25.0 & 8.0 & 0.9017 & 0.0261 & -3.29 & -3.08 & 1.14 & 0.000047 \\
\hline & & 20.0 & 0.6663 & 0.0224 & 2.59 & -0.32 & 1.10 & 0.000011 \\
\hline & & 30.0 & & 0.0182 & 4.59 & 1.13 & 1.1 & 0.000009 \\
\hline & & 60.0 & 0.35 & 0.0118 & 8.66 & 3.30 & 1.30 & 0.000006 \\
\hline & & 120.0 & 0.2037 & 0.0074 & 12.92 & 2.93 & 1.66 & 0.000004 \\
\hline & & 250.0 & 0.0964 & 0.0047 & 18.73 & -2.20 & 2.63 & 0.000002 \\
\hline & 100.0 & 25.0 & 0.8873 & 0.0117 & -2.35 & -2.30 & 1.11 & 0.000009 \\
\hline & & 60.0 & 0.5536 & 0.0136 & 4.34 & 1.61 & 1.12 & 0.000007 \\
\hline & & 90.0 & 0.3750 & 0.01 & 7.43 & 3.26 & 1.23 & 0.000008 \\
\hline & & 120.0 & 0.2628 & & 8.85 & 2.76 & 1.37 & 0.000007 \\
\hline & & & & & 10.54 & 2.12 & 1.54 & 0.000007 \\
\hline & & 200.0 & 0.1145 & 0.0096 & 11.30 & -1.15 & 1.83 & 0.000006 \\
\hline
\end{tabular}

\section{SENSITIVITY ANALYSIS}

Having studied the properties of the MLPDE when data come exactly from an IG distribution, a natural question to be asked is what will happen if the data does not follow an IG distribution, i.e., how sensitive is the estimator with respect to a departure from the IG assumption. There are many forms of departure from the IG assumption. We consider two popular forms and investigate the sensitivity of the MLPDE under each form of departure: a) the data is contaminated in the sense that a few observations arise from an IG distribution with a smaller or a larger mean while 
the majority follow the designated IG distribution, and b) the data is from another distribution. There are many other distributions that can be used to describe fatigue and lifetime data, such as lognormal, gamma, Weibull, etc.. However, in terms of failure pattern, the lognormal may be the one that is closest to the IG distribution in that both distributions are monotonic, increasing and then decreasing. The difference is that the lognormal failure rate converges to zero while that of IG converges to a constant as the 'time' approaches infinity.

The results given in Table 3 represent the case that the data is contaminated by either $5 \%$ or $10 \%$ on each side. The results show that the estimators are quite robust with respect to the contamination. They are less sensitive to contamination when the population is more skewed (the upper part of the table).

In practice, given a real data set, there may be more than one choice of distribution to fit. A goodness of fit test may help, but it usually fails to differentiate distributions of similar shape. We now investigate the sensitivity issue when data is from some other life distribution. Three popular life distributions, the lognormal, gamma and Weibull, are considered. Their pdfs are, $\frac{1}{\sqrt{2 \pi} \tau y} \exp \left[-\frac{1}{2 \tau^{2}}(\log y-\eta)^{2}\right]$, $\left(\frac{\tau}{\eta}\right)\left(\frac{y}{\eta}\right)^{\tau-1} \exp \left[-\left(\frac{y}{\eta}\right)^{\tau}\right]$, and $\frac{1}{\Gamma(\tau) \eta^{\tau}} y^{\tau-1} \exp \left(-\frac{y}{\eta}\right)$, respectively, $\eta>0, \tau>0$ and $y>0$.

Some results are given in Table 4. From the results we see that when data is generated from a lognormal distribution, the two estimators perform quite well, especially when data is not too skewed. The MLPDE again outperforms the MLE in terms of relative efficiency. However, when the data is generated from a distribution that has a different failure pattern, such as gamma and Weibull, the performance of the two estimators are poor, especially at the early stages. More simulation has been performed for both cases, including the one-sided contamination case. It is found the performance of the two estimators are worse in the one-sided contamination case.

\section{NUMERICAL EXAMPLES}


Table 3: Sensitivity under Contamination, $n=40, \mu=10$.

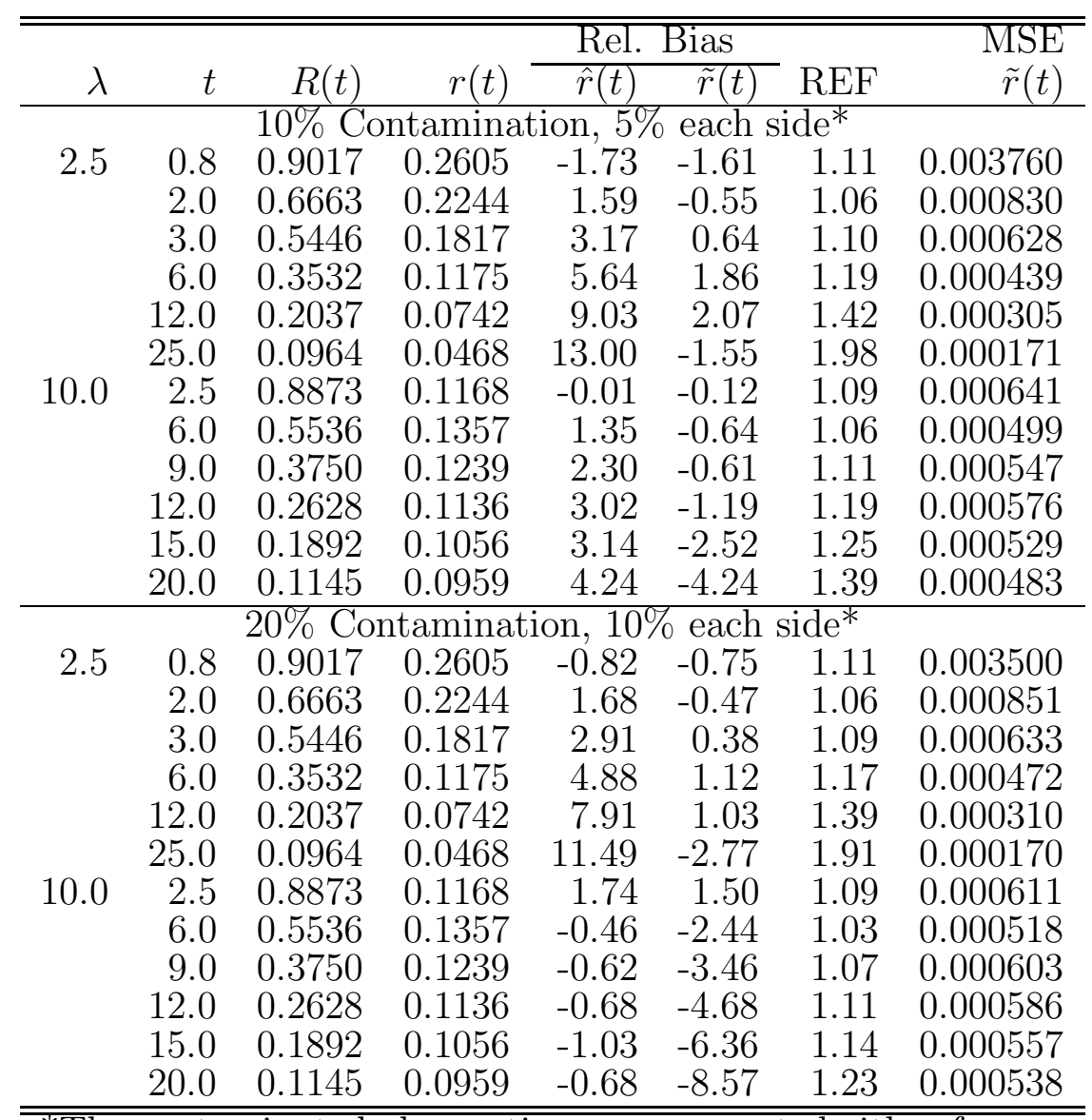

*The contaminated observations are generated either from $I G(\mu / 2, \lambda)$ or from $I G(2 \mu, \lambda)$

The numerical examples considered in Yang (1999) are considered again here to illustrate the failure rate estimation. The first two examples correspond to real data sets and the last corresponds a simulated data for the purpose of comparing the estimated failure rate with the true one. All three data sets are reproduced here for completeness. Again, our illustration concentrates on the most realistic case: both parameters unknown. The plots of the estimated failure rates in Figure 1 show that the MLE and MLPD agree well at the early stage, but start to move apart as the time increases. They can be significantly different at the tail area, especially when $n$ is small. The plot for the simulated data shows that the MLPD failure rate curve is 
Table 4: Sensitivity under Misspecification of Distribution, $n=30$

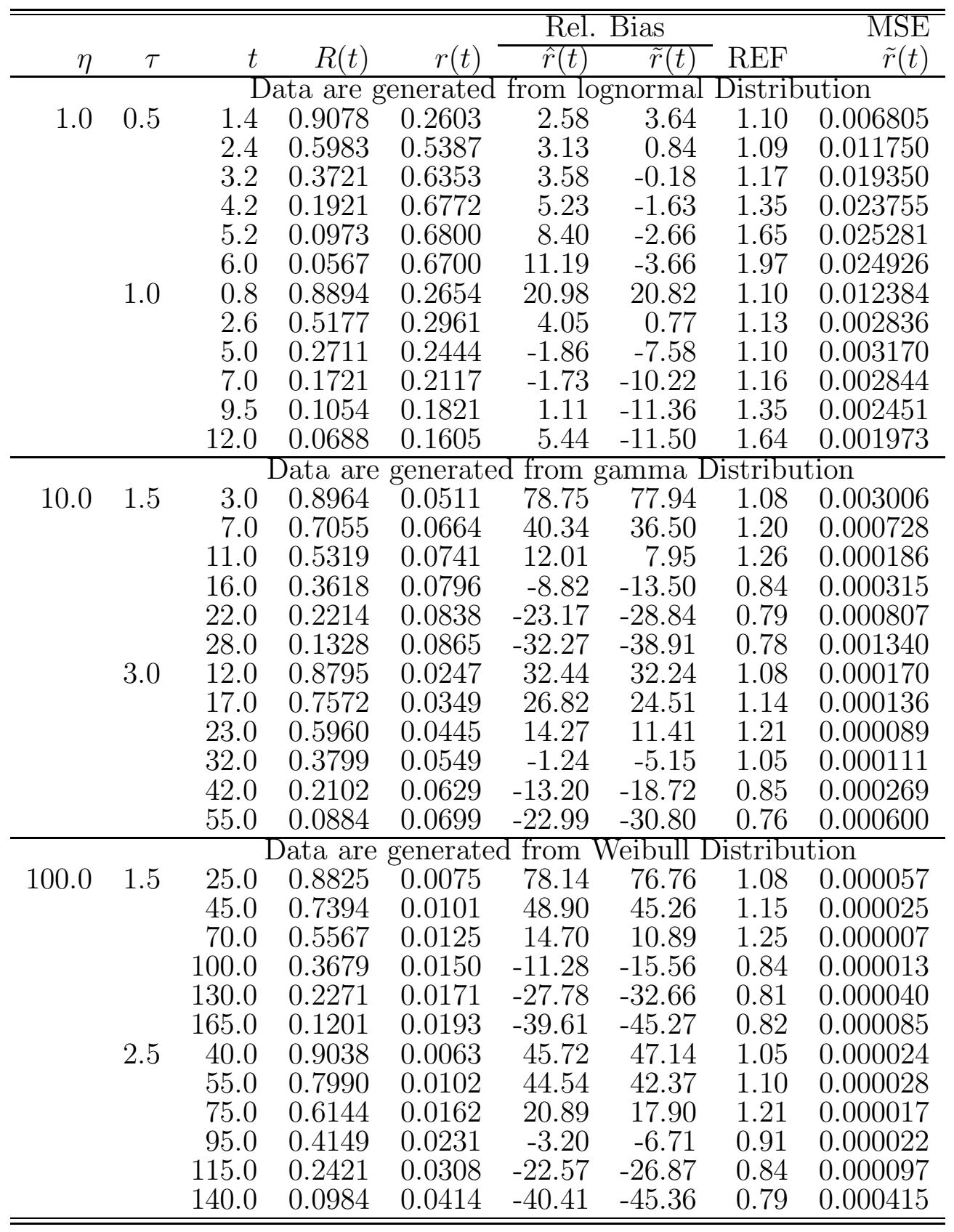


closer to the true curve than the MLE. The plots are obtained using MATHEMATICA 3.0 and a Pentium II $300 \mathrm{MHz}$ machine. It takes less than half a minute to finish this multiplot where each point of the plot involves numerical integrations.

Example 1. Fatigue lives (in hours) for 10 bearings tested on a certain tester: 152.7, 172.0, 172.5, 173.3, 193.3, 204.7, 216.5, 234.9, 262.6, 422.6. The MLEs are $\hat{\mu}$ $=220.48$ and $\hat{\lambda}=2708.86$. The data is slightly skewed to the right.

Example 2. 46 repair times (in hours) for an airborne communication transceiver: $.2, .3, .5, .5, .5, .5, .6, .6, .7, .7, .7, .8, .8,1.0,1.0,1.0,1.0,1.1,1.3,1.5,1.5$, 1.5, 1.5, 2.0, 2.0, 2.2, 2.5, 2.7, 3.0, 3.0, 3.3, 3.3, 4.0, 4.0, 4.5, 4.7, 5.0, 5.4, 5.4, 7.0, 7.5, 8.8, 9.0, 10.3, 22.0, 24.5. The MLEs for the parameters are $\hat{\mu}=3.61$ and $\hat{\lambda}=1.66$. The data is skewed to the right.

Example 3. Simulated Data. Fifteen observations are generated from an IG(1,1) population: $0.9144,0.2517,0.6506,0.9421,0.9112,0.2515,0.5057,0.9760$, $1.5257,0.5819,0.4591,0.6711,0.3103,0.3733,0.3696$. The resulted arithmetic and harmonic means are, respectively, $\bar{y}=0.6463$, and $\tilde{y}=0.4936$.

\section{Insert Figure 1 near here}

To conclude the article, we give a brief discussion. The method of applying MLPD to estimate reliability and failure rate seems promising and deserves further studies. There are other forms of predictive densities, such as the fiducial predictive density and the Bayesian predictive density, that can be used to serve the same purpose when the MLPD does not have a tractable mathematical form for a certain distribution.

Although the MLPD method outperforms the standard ML method in reliability and failure rate estimations, it is relatively straightforward to derive interval estimates for the reliability and failure rate functions using the latter approach. Alternatively, one may use the profile likelihood method (Kalbfleisch and Sprott, 1970) for these reliability studies, which has a further advantage of being Bartlett correctable. 


\section{Acknowledgements}

The authors are grateful to an anonymous referee for the constructive comments that lead to significant improvements on the article.

\section{References}

Chang, D.S. (1994) A note on constructing the confidence bounds of the failure rate for the inverse Gaussian distribution, Microelectron. Reliab., 34, 187-90.

Chhikara, R. S. and Folks, J. L. (1989) The Inverse Gaussian Distribution: Theory, Methodology and Applications, New York and Basel: Marcel Dekker, Inc.

Chhikara, R. S. and Guttman, I. (1982) Prediction limits for the inverse Gaussian distribution, Technometrics, 24, 319-324.

Kalbfleisch, J.D. and Sprott, D.A. (1970) Application of likelihood methods to models involving a large number of nuisance parameters (with discussion), J. Roy. Statist. Soc., B32, 175-208.

Lejeune, M. and Faulkenberry, G.D. (1982) A simple predictive density function, J. Amer. Statist. Assoc., 77 654-59.

Modarres, M. (1993) What Every Engineer Should Know About Reliability and Risk Analysis, New York, Basel and Hong Kong: Marcel Dekker, Inc.

Seshadri, V. (1993) The Inverse Gaussian Distribution: A Case Study in Exponential Families, Clarendon Press, Oxford.

Seshadri, V. (1998) The Inverse Gaussian Distribution: Statistical Theory and Applications, Springer-Verlag, New York, Inc.

Yang, Z.L. (1999) Maximum likelihood predictive densities for the inverse Gaussian distribution with applications to reliability and lifetime predictions. Microelectron. Reliab., 39, 1413-1421. 